\title{
Transtorno bipolar em crianças e adolescentes: critérios para diagnóstico e revisão de intervenções psicossociais
}

\author{
Renata Garcia de Almeida Moraes \\ Centro Universitário Filadélfia \\ Londrina, PR, Brasil \\ Márcia Cristina Caserta Gon \\ Universidade Estadual de Londrina \\ Londrina, PR, Brasil \\ Robson Zazula \\ Universidade Federal da Integração Latino Americana \\ Foz do Iguaçu, PR, Brasil
}

\begin{abstract}
Resumo
O transtorno bipolar é um transtorno mental crônico, recorrente e incapacitante. Entretanto, em crianças e adolescentes pode manifestar-se de forma diferente do padrão clássico descrito na idade adulta. Objetivou-se revisar e analisar estudos empíricos de intervenções psicossociais realizadas para o transtorno bipolar em crianças e adolescentes quanto aos seus objetivos, método, principais resultados e discussão. Foi realizada uma revisão sistemática de 21 artigos de intervenção psicossocial publicados entre 1999 e 2014. Constatou-se diversidade quanto às variáveis investigadas, tais como método, delineamento experimental, amostra, instrumentos e temas. Apesar disso, os estudos demonstraram eficácia em alguns aspectos estudados, em especial a comunicação e a informação aos pacientes e familiares. Sugere-se o desenvolvimento de pesquisas sobre transtorno bipolar em crianças e adolescentes, de modo a contribuir para a elaboração de ações ou programas de intervenção direcionados especificamente para esta população.
\end{abstract}

Palavras-chave: Transtorno bipolar; Infância; Adolescência; Intervenção psicossocial.

\section{Bipolar disorder in children and adolescents: criterions to diagnostic and review of psychosocial interventions}

\begin{abstract}
The bipolar disorder is a chronic, recurrent and incapacitating mental disease. Although with children and adolescents could occur differently than in adult age. The aim of this study was review and analyze empirical studies of psychosocial interventions for bipolar disorder with children and adolescents related to objectives, method, main results and data discussion. A systematic review was performance of 21 psychosocial intervention's articles, published between 1999 and 2014. The results showed diversity related to investigated variables, such as method, experimental design, sample, instruments and themes. Despite this, the studies demonstrated efficacy in some aspects investigated, specially communication and information to patients and their families. It suggests researches about bipolar disorder in children and adolescents to contribute for a large number of actions and intervention programs directly to this population.
\end{abstract}

Keywords: Bipolar disorder; Childhood; Adolescence; Psychosocial intervention.

\section{Trastorno bipolar en niños y adolescentes: criterios para el diagnóstico y revisión de las intervenciones psicosociales}

\section{Resumen}

El trastorno mental crónico es un trastorno mental crónico, recurrente y incapacitante. Sin embargo, en los niños y adolescentes pueden manifestar patrón diferente del descrito en la edad adulta. El objetivo de este estudio es revisar y analizar los estudios empíricos de las intervenciones psicosociales para el trastorno bipolar en niños y adolescentes cuanto a los objetivos, método, principales resultados y discusión. Una revisión sistemática analizó 21 intervenciones psicosociales los artículos publicados entre 1999 y 2014. Se obtuvo diversidad cuanto a las variables investigadas, como el método, el delineamiento experimental, amuestra, instrumentos y temas. Sin embargo, los estudios demostraran eficacia en algunos de los aspectos investigados, en especial la comunicación y la información para los pacientes y familiares. Se sugiere el desarrollo de estudios sobre el trastorno bipolar en niños y adolescentes, de modo a contribuir para la elaboración de acciones y programas de intervención direccionados para esta población.

Palabras-clave: Trastorno bipolar; Infancia; Adolescencia; Intervención psicosocial. 
Anteriormente conhecida como psicose maníacodepressiva, o transtorno bipolar (TB) é considerado pela psiquiatria como um transtorno mental com quadro crônico, recorrente e incapacitante (APA, 2013). Representa um grande problema de saúde com graves consequências sociais e econômicas devido, sobretudo, a não adesão ao tratamento (Colom \& Vieta, 2004; Leclerc, Mansur, \& Brietzke, 2013). O TB caracteriza-se pelas condições de mania (elação com comportamento desinibido e hiperativo) e depressão (mudança extrema no estado de ânimo e comportamento; APA, 2013; Birmaher, 2013; Grande, Berk, Birmaher, \& Vieta, 2015).

De acordo com a quinta edição do Manual Estatístico e Diagnóstico de Doenças Mentais (DSM-V; APA, 2013), a fase de mania pode ser entendida como um período distinto de humor anormal e continuamente elevado, expansível ou irritável. É uma fase de muita "energia" que pode ser perturbadora e prejudicial para o paciente e para aqueles com quem convive. Durante tais episódios pode haver alterações como: diminuição ou ausência de sono, aumento na quantidade de exercícios, forte impulso para ser mais ativo social ou sexualmente e recusa em comer e beber. Por sua vez, a fase de depressão caracteriza-se pelo humor triste, vazio ou irritável, que é acompanhado de diversas alterações fisiológicas que influenciam diretamente no funcionamento do indivíduo. Dentre as principais alterações, destacam-se: diminuição da atividade motora, depressão do humor, melancolia e dificuldade de concentração.

Em relação à incidência do $\mathrm{TB}$, de acordo com o DSM-V (APA, 2013), mais de 1\% da população é afetada, independentemente da nacionalidade, origem étnica ou condições socioeconômicas. Segundo Grande et al. (2015), estima-se que em todo o mundo a prevalência é de $0,6 \%$ para TB do tipo I, $0,4 \%$ para TB tipo II e 2,4\% para TB não especificado. Dados empíricos demonstram que a idade média para a manifestação do primeiro episódio maníaco ou depressivo é 20 anos, ou seja, inicia-se predominantemente na idade adulta (Morken, Vaaler, \& Folden, 2009). Porém, é crescente o número de pesquisas que têm constatado a presença de sintomas e a manifestação do TB em idades inferiores aos 20 anos (e.g., Brotman et al., 2006; Duzetzina et al., 2012; Goldstein, 2012; Leibenluft, 2011; Pavuluri, Birmaher, \& Naylor, 2005; Syed, Sheriff, McGorry, Cotton, \& Yung, 2015; Van Meter, Burke, Kowatch, Finding, \& Youngstrom, 2016).

Não existem dados conclusivos sobre a prevalência do TB em crianças e adolescentes. Resultados de um estudo epidemiológico realizado por West e Pavuluri (2009) mostraram que esta é de 1\% em adolescentes de
14 e 18 anos. Um estudo de metanálise, conduzido por Van Meter, Moreira e Youngstrom (2011), encontrou que a prevalência do $\mathrm{TB}$ em crianças e adolescentes era de 1,8\%. De acordo com o DSM-V (APA, 2013), nos Estados Unidos a prevalência de TB em crianças e adolescentes varia entre 0,0 e $0,6 \%$ da população. Contudo, esse número pode ser maior, pois quando são investigados pacientes adultos, $60 \%$ relatam sintomas antes dos 20 anos e entre $10 \%$ a $20 \%$ dos pacientes relatam os primeiros sintomas antes dos 10 anos de idade.

Assim como com adultos, o TB em crianças e adolescentes é considerado grave e está associado a mudanças do humor. Os principais sintomas incluem dificuldade em seguir regras (que resulta em problemas emocionais, relacionamento social e funcionamento acadêmico), desesperança e poucas habilidades de coping. West e Pavuluri (2009) salientam ainda que uma contingência presente na vida desses indivíduos é a ocorrência de eventos negativos e stress crônico.

Além disso, o TB em crianças e adolescentes pode apresentar-se de modo diferente do padrão descrito na idade adulta. Crianças geralmente apresentam ciclagem rápida, com quatro episódios de humor por ano, bem como alterações bruscas diárias de humor (i.e., uma vez ao dia), sintomas depressivos mistos (i.e., muitas vezes maníacos, na forma de irritabilidade) e evolução insidiosa e crônica desde a infância (Phillips \& Kupfer, 2013). No DSM-V (APA, 2013), são adotados os critérios gerais para diagnóstico do TB em adultos, com adição de dois aspectos: humor depressivo irritável e dificuldade em ganhar peso. Ainda, se deve comparar os comportamentos apresentados pela criança com aqueles que são esperados para a etapa do desenvolvimento na qual ela se encontra. De acordo com a Classificação Estatística Internacional de Doenças (CID-10; OMS, 1992), os critérios para diagnóstico de TB em crianças e adolescentes são: irritabilidade, agressividade e estados de humor misto.

Outra dificuldade para diagnosticar TB em crianças e adolescentes é a possível ocorrência de comorbidades (Phillips \& Kupfer, 2013). Segundo o CID-10, observa-se comorbidade de $90 \%$ do TB em crianças e adolescentes com o transtorno de déficit de atenção (TDAH). De acordo com o DSM-V (APA, 2013), o TB em crianças e adolescentes pode possuir comorbidade de até $60 \%$ com outras doenças mentais (e.g. TDAH), até 75\% com transtornos de ansiedade, até 37\% com o uso de substâncias (e.g., álcool, drogas lícitas ou ilícitas) e até $14 \%$ podem apresentar pelo menos um episódio de distúrbio alimentar ao longo de sua vida. Pesquisas evidenciam nesta popu- 
lação altas taxas de comorbidade como transtorno de déficit de atenção e hiperatividade (TDAH), comportamento opositor e transtorno de ansiedade (e.g., Crump, Sundquist, Winkleby, \& Sundquist, 2013; Frías, Palma, \& Farriols, 2015; Miller, Chang, \& Ketter, 2013; Phillips \& Kupfer, 2013; Serrano, Ezpeleta, Alda, Matalí, \& San, 2011; Yen et al., 2015; Young et al., 2013). Um estudo realizado por Weller, Kloos, Weisbrot e Weller (2006) indicou também que crianças e adolescentes têm maiores características psicóticas e um curso mais grave do TB do que indivíduos adultos.

Na tentativa de resolver algumas discordâncias entre os pesquisadores sobre a natureza, definição do TB em crianças e adolescentes, e critérios para diagnosticálo, o DSM-V (APA, 2013), traz que crianças com instabilidade de humor podem ser incluídas em uma das seguintes categorias: TB do tipo I (i.e., episódios de mania e depressão, que são diagnosticados com base nos episódios maníacos), TB tipo II (i.e., ocorrência de um ou mais episódios depressivos maiores e elo menos um episódio hipomaníaco), transtorno ciclotímico (i.e., períodos de hipomania e depressão que não atendem aos critérios para episódios de mania, hipomania ou depressão maior, por pelo menos um ano) e o TB não especificado (i.e., instabilidade de humor, mas que não satisfazem os critérios do manual. Portanto, essas últimas geralmente recebem o diagnóstico de TB não especificado). Apesar de existirem controvérsias quanto à forma de diagnosticar o $\mathrm{TB}$ em crianças e adolescentes, estratégias de intervenções farmacológicas e não farmacológicas para o tratamento dessa população têm sido alvo de investigações por meio de pesquisas empíricas (e.g., Angst, 2013; Axelson et al., 2015; Phillips \& Kupfer, 2013; Severus \& Bauer, 2013).

Muitos medicamentos indicados para pacientes adultos com TB são prescritos para crianças e podem trazer melhoras clínicas, reduzir os fatores desestabilizadores do humor e aumentar estratégias estabilizadoras. $\mathrm{O}$ frequente abandono do tratamento pode ser por desconhecimento do problema ou a preferência pelo humor eufórico (Belzeaux et al., 2015). Embora o tratamento farmacológico esteja associado a prevenções de recaídas e ao menor risco de recidivas, $60 \%$ dos pacientes que o fazem têm recaídas nos dois anos após um episódio agudo, e pelo menos $50 \%$ experimentam sintomas relacionados com a oscilação do humor entre episódios (Miklowitz, George, Richards, Simoneau, \& Suddath, 2003). Além disso, após o controle dos sintomas, os pacientes podem apresentar prejuízos em relação ao desempenho acadêmico e relacionamentos. Tal condição indica limitações da farmacoterapia e, assim, a recomendação para intervenções psicossociais (Prien \& Rush, 1996).

Apesar de intervenções psicossociais serem recomendadas e necessárias para pacientes com TB, Ramires, Benetti, Silva e Flores (2009) enfatizaram que a falta de reconhecimento da importância da saúde mental na infância e adolescência pode acarretar na não identificação precoce dos sintomas e oportunidades de tratamento adequado. Em crianças e adolescentes com TB este quadro não é diferente, sobretudo porque o diagnóstico é complicado, havendo divergências quanto a sua realização (Phillips \& Kupfer, 2013).

Pesquisas sobre intervenções psicossociais existentes se mostram úteis no estudo do TB de crianças e adolescentes uma vez que contribuem para o seu aprimoramento tecnológico, para um maior conhecimento da problemática do TB nessa população e atuam como estratégias que auxiliam o tratamento farmacológico (Miklowitz, Schneck et al., 2014). Diferentes estratégias de intervenção psicossocial são utilizadas por grupos de pesquisadores da área, destacando-se como exemplos, a denominada Grupo Psicoeducacional Multifamiliar (Multi-family Psychoeducational Group - MFPG; Fristad, Goldberg-Arnold, \& Gavazzi, 2002); a Psicoeducação Familiar Individual (Individual Family Psychoeducation - IFP; Fristad, Goldberg-Arnold, \& Gavazzi, 2002); o Tratamento para Adolescentes Focado na Família (Family-Focused Treatment for Adolescents - FFT-A; Miklowitz et al., 2004); a Terapia Dialética Comportamental para Adolescentes (Dialectical Behavior Therapy for Adolescents; Goldstein, Axelson, Birmaher \& Brent, 2010), a Terapia Cognitivo-Comportamental Focada na Criança e na Família (Child and Family-Focused Cognitive-Behavioral Therapy - CFF-CBT; Pavuluri et al., 2004), a Terapia Periódica Interpessoal e Social (Interpersonal and Social Rhythm Therapy, ISRT; Goldstein et al., 2014), dentre outras.

Embora as intervenções psicossociais mencionadas tenham por base a psicoeducação, a hipótese é que não parece haver uma sistematização metodológica entre as pesquisas realizadas que as utilizam e os resultados obtidos são diversos. Por esta razão, objetivou-se com este trabalho revisar e analisar estudos empíricos de intervenções psicossociais realizadas para o TB em crianças e adolescentes quanto aos seus objetivos, método, principais resultados e discussão. Além disso, devido a sua importância, uma breve análise sobre a questão dos critérios para diagnosticar o TB nesta população será apresentada como parte da discussão do presente trabalho. 


\section{Método}

Foi realizada uma pesquisa eletrônica nas seguintes bases de dados: (a) Academic Search Premier, (b) MEDLINE/PubMed, (c) MEDLINE/ via Bireme, (d) MEDLINE/via OvidAcademic, (e) Web of Science, (f) SCOPUS (Elsevier), (g) Lilacs e (h) PsycINFO. As palavras ou expressões-chave utilizadas foram: bipolar disorder, child, adolescents, comorbidity, treatment, assessment, psychoeducational intervention, psychosocial intervention, psychological intervention, education program, therapeutic education.

Foram incluídos artigos que apresentaram relatos originais de pesquisas empíricas de intervenção psicossocial com crianças e/ou adolescentes diagnosticados com TB, publicados entre os anos de 1999 e 2014, em inglês, espanhol e português. Os mesmos critérios para seleção dos resumos foram adotados em todas as bases de dados consultadas. Foram excluídos os resumos repetidos e/ou incompletos e as pesquisas teóricas, revisão, meta-análises, estudos empíricos apenas de diagnóstico ou avaliação e intervenções farmacológicas.

Após a coleta dos dados foi realizada a leitura dos resumos que atenderam aos critérios de inclusão. A segunda etapa constituiu-se da seleção dos artigos empíricos de intervenção psicossocial, totalizando 21. Por fim, na terceira etapa realizou-se a busca dos artigos na íntegra, selecionados na etapa anterior, para sua posterior categorização e análise.

A qualidade científica das pesquisas selecionadas foi analisada a partir das seguintes categorias: (a) objetivos da pesquisa, (b) amostra (populaçãoalvo e idade dos participantes), (c) delineamento do estudo e utilização de grupo controle, (d) follow-up, (e) instrumentos de medidas utilizados, (f) temas abordados nas intervenções e (g) principais resultados.

\section{Resultados}

Foram recuperados 21 artigos que atendiam aos critérios de inclusão estabelecidos. Quanto aos objetivos dos estudos selecionados, todos avaliaram a eficácia de intervenções que continham componentes psicoeducacionais, a partir de diferentes perspectivas. Dentre os estudos revisados, o principal objetivo destes foi avaliar a intervenção no comportamento da família e dos pacientes com TB e os efeitos da intervenção nos sintomas do TB durante e após as intervenções. Houve ainda estudos com o objetivo de avaliar o efeito da intervenção na redução de peso.
Em relação ao número de participantes dos estudos, houve variação entre 1 e 463 indivíduos. Dos 21 estudos analisados, em 12 participaram apenas crianças e/ou adolescentes com TB e em nove participaram os pais e/ou cuidadores. A idade dos participantes variou entre cinco e mais de 65 anos. Seis desses estudos foram realizados com participantes com idade mínima de oito anos. Nenhum dos estudos avaliados informou a idade dos pais.

Quanto aos critérios para participação nos estudos, exigiu-se que a criança e/ou adolescente tivesse o diagnóstico dos transtornos bipolar. Em seis dos estudos também foi necessário que a criança apresentasse pelo menos um episódio maníaco com exaltação e grandiosidade, misto, hipomaníaco ou depressivo em até seis meses anteriores à intervenção. Em seis dos estudos foi exigida a participação efetiva de um dos responsáveis. Em quatro deles as crianças deveriam apresentar estabilidade em relação aos efeitos farmacológicos e o interesse em permanecer submetendo-se ao tratamento medicamentoso. Em dois foi aplicado um teste de quociente de inteligência e exigida pontuação mínima para participação, sendo que um deles exigiu também uma determinada pontuação nas escalas Young Mania Rating Scale (YMRS) ou Childrens Depression Rating Scale - Revised (CDRS-R).

Quanto aos delineamentos de pesquisa, constatouse que oito dentre os 21 estudos foram individuais e quase-experimentais, sendo que, todos realizaram testes pré e pós-intervenção. Nove foram estudos experimentais: três realizaram linha de base múltipla entre grupos, três foram randomizados e controlados, quatro utilizaram grupo controle, sendo dois longitudinais e multicêntricos. Os quatro restantes foram estudos de caso e, destes, apenas um utilizou medidas de pré e pós-testes. Foram realizados follow-up dos resultados em 14 estudos: sete fizeramno após decorrido um ano da intervenção, quatro após seis meses e três após dois meses. Tais informações estão descritas na Tabela 1.

Quanto às medidas utilizadas, para avaliação pré e pós-intervenção, estas totalizaram 37 nos 21 estudos, sendo seis as mais frequentemente utilizadas: Young Mania Rating Scale (YMRS) em 12 estudos; Kiddie Schedule for Affective Disorders and Schizophrenia, Present and Lifetime Version (K-SADS-PL) em oito estudos; Child's Global Assessment Scale (CGAS) em seis estudos; Expressed Emotion Adjective Checklist (EEAC) em cinco estudos; Children's Interview for Psychiatric Syndromes (CHIPs) em cinco estudos e Children's Depression Rating Scale-Revised (CDRS-R) em cinco estudos e outros três estudos utilizaram 
TABELA 1

Descrição da amostra, delineamento de pesquisa, presença de grupo controle e realização de follow-up nos estudos revisados

\begin{tabular}{|c|c|c|c|c|c|c|}
\hline \multirow[b]{2}{*}{ Estudo } & \multicolumn{3}{|c|}{ Amostra } & \multirow[b]{2}{*}{ Delineamento } & \multirow{2}{*}{$\begin{array}{l}\text { Grupo } \\
\text { Controle }\end{array}$} & \multirow[b]{2}{*}{ Follow-up } \\
\hline & Público-alvo & $\begin{array}{l}\text { Quant. } \\
\text { (n) }\end{array}$ & $\begin{array}{l}\text { Idade } \\
\text { (anos) }\end{array}$ & & & \\
\hline Danielson et al. (2004) & Adolescentes & 1 & 13 & Estudo de caso & Não & Sim \\
\hline Fagiolini et al. (2009) & $\begin{array}{l}\text { Adolescentes } \\
\text { Adultos } \\
\text { Idosos }\end{array}$ & 463 & $\begin{array}{c}12-17 \\
18-64 \\
>65\end{array}$ & $\begin{array}{l}\text { Longitudinal, multicêntrico } \\
\text { randomizado e controlado. }\end{array}$ & Sim & Não \\
\hline Feeny et al. (2006) & Crianças e pais & 16 & $10-17$ & Randomizado e controlado & Sim & Sim \\
\hline Fristad (2006) & Crianças e pais & 20 & $8-11$ & Randomizado e controlado & Sim & Sim \\
\hline Fristad et al.(2002) & Crianças e pais & 84 & $\begin{array}{c}\bar{X}=10,1 \\
(8-11)\end{array}$ & Randomizado e controlado & $\operatorname{Sim}$ & $\operatorname{Sim}$ \\
\hline \multirow{4}{*}{$\begin{array}{l}\text { Fristad et al. (2003) } \\
\text { (Estudos I, II, III e IV) }\end{array}$} & Pais & 25 & & Randomizado e controlado & Não & Sim \\
\hline & Crianças e pais & 12 & $8-11$ & Quase-experimental & Não & Não \\
\hline & Famílias & 35 & & $\begin{array}{l}\text { Randomizado e controlado com linha } \\
\text { de base múltipla entre grupos. }\end{array}$ & Não & $\operatorname{Sim}$ \\
\hline & Famílias & 20 & & Randomizado e controlado & Não & Não \\
\hline Fristad et al. (2007) & Criança & 1 & 12 & Estudo de caso & Não & Não \\
\hline Fristad et al. (2009) & Crianças & 165 & $8-12$ & Randomizado e controlado & $\operatorname{Sim}$ & $\operatorname{Sim}$ \\
\hline Goldstein et al. (2010) & Adolescentes e pais & 10 & $\begin{array}{l}\bar{X}=15,8 \\
(14-18)\end{array}$ & Quase-experimental & Não & Não \\
\hline Goldstein et al. (2011) & Adolescentes & 1 & 15 & Estudo de caso com pré e pós- testes & Não & Não \\
\hline Goldstein et al. (2014a) & Crianças e adolescentes & 69 & $7-13$ & Grupo & Não & $\operatorname{Sim}$ \\
\hline Goldstein et al. (2014b) & Adolescentes & 10 & $\begin{array}{c}\bar{X}=16,9 \\
13-18\end{array}$ & Grupo & Não & Sim \\
\hline Hlastala et al. (2010) & Adolescentes e familiares & 12 & $\begin{array}{l}\bar{X}=16,5 \\
(12-18)\end{array}$ & Quase-experimental & Não & Não \\
\hline Jairam et al. (2004) & Crianças & 25 & $9-16$ & Quase-experimental & Não & Sim \\
\hline Leffler et al. (2010) & Crianças/pais & 2 & $8-12$ & Estudo de caso & Não & Não \\
\hline Miklowitz et al. (2004) & Adolescentes e pais & 52 & $\begin{array}{l}\bar{X}=14,8 \\
(13-17)\end{array}$ & Quase-experimental & Não & $\operatorname{Sim}$ \\
\hline Miklowitz et al. (2008) & Adolescentes, irmãos e pais & 58 & $\bar{X}=14,5$ & Randomizado e controlado & Não & Sim \\
\hline Miklowitz et al. (2011) & Crianças e/ou adolescentes & 13 & $\begin{array}{c}\bar{X}=13,4 \\
(9-18)\end{array}$ & Quase-experimental & Não & $\operatorname{Sim}$ \\
\hline Miklowitz et al. (2014) & Crianças e adolescentes & 40 & $9-17$ & Grupo randomizado & Não & Sim \\
\hline Pavuluri et al. (2004) & Crianças e pais & 34 & $\begin{array}{c}11,3 \\
(5-17)\end{array}$ & Quase-experimental & Não & Não \\
\hline West et al. (2009) & Crianças e Famílias & 52 & $6-12$ & Quase-experimental & Não & Não \\
\hline
\end{tabular}

outros instrumentos. Quanto aos temas abordados nas intervenções, observa-se que as informações básicas sobre o TB foram trabalhadas em todos os estudos. Os temas abordados e os respectivos estudos estão descritos na Tabela 2.

Quanto aos resultados, apesar dos objetivos serem semelhantes e os delineamentos diversos, 18 dos 21 estudos constataram após a intervenção uma redução significativa dos sintomas do TB em crianças e adolescentes. Vale ressaltar que nenhuma das pesquisas apontou para resultados adversos após a intervenção.
Dentre os principais resultados obtidos, 15 descreveram a redução dos sintomas do TB (e.g., instabilidade de humor, irritabilidade e apatia), cinco relataram que pais e/ou mães passaram a se comportar de maneira mais adequada frente ao TB de seus filhos, três descreveram melhorias no relacionamento interpessoal e aumento no apoio familiar e social. Os estudos constataram ainda que as intervenções proporcionaram diminuição de peso, melhorias no rendimento escolar, mudanças nos sentimentos e desenvolvimento de estratégias de coping. 
TABELA 2

Temas abordados durante as intervenções dos estudos revisados

\begin{tabular}{|c|c|}
\hline Temas abordados & Estudos \\
\hline $\begin{array}{l}\text { Informações sobre adesão ao tratamento } \\
\text { medicamentoso (14) }\end{array}$ & $\begin{array}{l}\text { Danielson et al. (2004); Fagiolini et al. (2009); Fristad et al. (2002); Goldstein et al. (2010); } \\
\text { Goldstein et al. (2014a); Goldstein et al. (2014b); Hlastala et al. (2010); Jairam et al. (2004); } \\
\text { Leffler et al. (2010); Miklowitz et al. (2004); Miklowitz et al. (2008); Miklowitz et al. (2014); } \\
\text { West et al. (2009); West et al. (2014). }\end{array}$ \\
\hline Treino em resolução de problemas (11) & $\begin{array}{l}\text { Danielson et al. (2004); Feeny et al. (2006); Goldstein et al. (2010); Goldstein et al. (2014a); } \\
\text { Leffler et al. (2010); Miklowitz et al. (2004); Miklowitz et al. (2008); Miklowitz et al. (2011); } \\
\text { Pavuluri et al. (2004); West et al. (2009); West et al. (2014). }\end{array}$ \\
\hline Manejo dos sintomas (11) & $\begin{array}{l}\text { Fristad et al. (2007); Goldstein et al. (2010); Goldstein et al. (2014a); Goldstein et al. (2014b); } \\
\text { Jairam et al. (2004); Leffler et al. (2010); Miklowitz et al. (2004); Miklowitz et al. (2014); } \\
\text { Pavuluri et al. (2004); West et al. (2009); West et al. (2014). }\end{array}$ \\
\hline $\begin{array}{l}\text { Hábitos saudáveis (e.g., dietas, exercícios físicos, } \\
\text { sono e higiene) (6) }\end{array}$ & $\begin{array}{l}\text { Fagiolini et al. (2009); Fristad (2006); Fristad et al. (2003); Goldstein et al. (2011); } \\
\text { Hlastala et al. (2010); Leffeler et al. (2010). }\end{array}$ \\
\hline Desenvolvimento de habilidades sociais $(8)$ & $\begin{array}{l}\text { Danielson et al. (2004); Feeny et al. (2006); Goldstein et al. (2014); Miklowitz et al. (2008); } \\
\text { Miklowitz et al. (2014); Pavuluri et al. (2004); West et al. (2009); West et al. (2014). }\end{array}$ \\
\hline $\begin{array}{l}\text { Identificação e modificação de sentimentos e/ou } \\
\text { de pensamentos que influenciem na estabilidade } \\
\text { e melhoria do humor (7) }\end{array}$ & $\begin{array}{l}\text { Danielson et al. (2004); Fristad et al. (2009); Goldstein et al. (2010); Goldstein et al. (2014a); } \\
\text { Jairam et al. (2004); West et al. (2009); West et al. (2014). }\end{array}$ \\
\hline $\begin{array}{l}\text { Identificação de eventos desencadeantes e manejo } \\
\text { de stress (6) }\end{array}$ & $\begin{array}{l}\text { Feeny et al. (2006); Goldstein et al. (2014a); Goldstein et al. (2014b); Miklowitz et al. (2004); } \\
\text { Miklowitz et al. (2008); Miklowitz et al. (2011). }\end{array}$ \\
\hline $\begin{array}{l}\text { Informações sobre os efeitos colaterais da } \\
\text { medicação (4) }\end{array}$ & Fagiolini et al. (2009); Goldstein et al. (2011); Hlastala et al. (2010); Jairam et al. (2004). \\
\hline Relacionamento familiar (conflitos) (5) & $\begin{array}{l}\text { Fristad et al. (2002); Goldstein et al. (2014a); Miklowitz et al. (2008); } \\
\text { Miklowitz et al. (2011); Miklowitz et al. (2014). }\end{array}$ \\
\hline $\begin{array}{l}\text { Sinais precoces de recaídas (e.g., eventos } \\
\text { desencadeantes) (4) }\end{array}$ & Fagiolini et al. (2009); Goldstein et al. (2014b); Jairam et al. (2004); Miklowitz et al. (2011). \\
\hline Abuso de substâncias (i.e., álcool e/ou drogas) (2) & Danielson et al. (2004); Feeny et al. (2006). \\
\hline Estratégias para redução da emoção expressa (2) & Goldstein et al. (2014a); Jairam et al. (2004). \\
\hline
\end{tabular}

\section{Discussão}

A partir dos resultados da presente revisão, observouse que há uma diversidade das variáveis investigadas, métodos e instrumentos de avaliação utilizados, o que contribui para a impossibilidade de propor uma generalização dos resultados. Apesar disso, os estudos demonstraram eficácia em alguns aspectos, tais como, a redução dos sintomas do TB (e.g., Miklowitz et al., 2008; Fristad et al., 2009; Fristad, 2006; Fristad et al., 2002; Miklowitz et al., 2004; Miklowitz et al., 2011), comportamentos mais adequados dos pais frente ao TB de seus filhos (e.g., Fristad et al., 2003; Fristad, 2006); melhora do relacionamento familiar (e.g., Fristad, 2006; Fristad et al., 2002); ganho de experiência, aumento do conhecimento dos pais em relação à doença e aumento do apoio familiar e social (e.g., Fristad et al., 2003; Fristad et al., 2002). Estes resultados se mantiveram após o follow-up.

Dentre as estratégias psicoeducacionais que foram utilizadas nestas pesquisas, destacaram-se a comunicação e a informação como principais componentes da adesão ao tratamento do transtorno. Para diversos autores (e.g., Geddes \& Miklowitz, 2013; Gibson et al., 2013; Miklowitz et al., 2014; Reinares, Sanchéz-Moreno, \& Fountoulakis, 2013), estes componentes favorecem a melhor aceitação do tratamento e/ou a adaptação ao transtorno pelo paciente e pela sua família. Todos os estudos revisados utilizaram estes componentes nas intervenções e demonstram sua importância também para atendimento de crianças e adolescentes.

Constatou-se que na maioria dos estudos revisados, os participantes eram crianças acima de seis anos. Conforme West et al. (2009), crianças acima dessa idade são frequentemente alvos de pesquisas de intervenções psicossociais. Isso ocorre, segundo o mesmo autor, uma vez que elas são consideradas cognitivamente desenvolvidas a ponto de beneficiarem-se deste tipo de intervenção. Além do mais, crianças nesta faixa etária já apresentariam também repertório de comportamentos de autocuidado e, conforme aspectos culturais, 
passariam a exercer uma função mais ativa em relação aos cuidados com sua saúde, tornam-se responsável pela realização de tarefas relacionadas ao tratamento e apresentam comportamentos de prevenção e promoção da saúde (Zazula \& Gon, 2014).

Contudo, poucos estudos empíricos de intervenção psicossocial para o TB em crianças e adolescentes foram localizados ao longo de um período de 15 anos. Uma possível explicação para este resultado é o ineditismo da área e, em virtude disso, o conceito TB infantil ser ainda inconsistente e controverso. De acordo com Phillips e Kupfer (2013), o TB em crianças e adolescentes é muitas vezes de difícil diagnóstico, uma vez que os critérios para avaliação do transtorno nessa faixa etária, tanto no contexto clínico quanto na pesquisa, ainda não estão padronizados. Isso pode dificultar o diagnóstico e a definição de propostas de intervenção que possam ser mais efetivas e que beneficiem a maioria dos pacientes e suas famílias. Segundo Serrano et al. (2011), a apresentação clínica de mania em crianças, a sobreposição dos sintomas com outras doenças mentais, as variações no método (e.g., idades, escala de classificação) e manuais com diferentes critérios diagnósticos são variáveis que dificultam diagnosticar o TB nesta população.

Por muito tempo, o TB em crianças e adolescentes foi subdiagnosticado, mal reconhecido e confundido com outros quadros psicopatológicos (i.e., Frías et al., 2015; Miller et al., 2013; Phillips \& Kupfer, 2013; Pacheco et al., 2014; Rocha \& Fontes-Neto, 2007; Yen et al., 2015). Atualmente, para diagnosticá-lo têm sido utilizados com mais frequência a maioria dos critérios gerais do DSM-V (APA, 2013), que descrevem o TB em adultos. Entretanto, há argumentos desfavoráveis em relação aos critérios do manual para avaliação e diagnóstico do TB nesta faixa etária porque as alterações de humor características se manifestam a partir da adolescência, tornando contraindicado o seu uso com crianças (Birmaher, 2013).

Além disso, todas as crianças e adolescentes certamente apresentam alterações de humor características de sua faixa etária durante o seu desenvolvimento. No entanto, como em todos os transtornos, tais variações se tornam clinicamente significativas apenas quando ocorrem a ponto de causar comprometimento funcional (Apps et al., 2008). Dependendo da idade, crianças podem apresentar alguns comportamentos topograficamente semelhantes àqueles descritos como sintomas de TB, mas que não são (e.g., choro excessivo ou irritabilidade podem ser apresentados devido a uma dificuldade de expressão verbal decorrente do processo de aprendizagem). Assim, o autor chama atenção para a diferença entre o repertório comportamental de adultos e crianças, salientando que nos manuais diagnósticos os critérios diagnósticos estão relacionados com comportamentos do adulto e, por essa razão, faz-se contraindicado para avaliação de crianças e/ou adolescentes (Phillips \& Kupfer, 2013).

Inúmeras controvérsias, portanto, são observadas nos critérios para se diagnosticar o TB em crianças e adolescentes. De acordo com Duffy (2007), para se constatar a idade exata de início da doença, não se deve apenas considerar os sintomas apresentados. É necessária também uma investigação detalhada sobre a evolução clínica da doença e o histórico familiar. Youngstrom, Freeman e Jenkins (2009) destacam que o TB em crianças e adolescentes é incomum e complexo porque pode mudar drasticamente à medida que os indivíduos transitam por diferentes episódios ao longo da vida. Assim, somente após verificar tanto os episódios presentes quanto do passado é possível realizar o diagnóstico com precisão.

Os critérios diagnósticos propostos pelo DSM-V (APA, 2013) para o TB não reconhecem a presença de comorbidades com transtornos frequentes na infância e adolescência, destacando apenas humor depressivo irritável e dificuldade em ganhar peso. Tal fator limita o uso do manual para diagnóstico em idade precoce. A ciclagem rápida, humor misto e a natureza episódica dos sintomas em crianças e adolescentes com TB podem não atender também os critérios de intensidade ou duração dentro das classes presentes do DSM-V (e.g., tipo I, tipo II; APA, 2013). Em virtude disso, ao se avaliar o TB em crianças e adolescentes, faz-se necessário avaliar as comorbidades, além de realizar entrevistas estruturadas, objetivas e abrangentes com a descrição dos sintomas, fato que pode minimizar o viés diagnóstico.

Com base nestas dificuldades mencionadas para a realização de um diagnóstico confiável do TB em crianças e adolescentes é importante: (a) utilizar escalas de avaliação direcionadas à população infantil, (b) conhecer a história de vida do paciente e de sua família além de (c) empregar os manuais diagnósticos (Carr, 2009). Esta sistematização seria importante para (a) a realização de um diagnóstico clínico mais confiável de indivíduos com suspeita do transtorno que procuram os serviços de saúde mental público ou privado e, (b) possibilitaria a adoção de critérios mais precisos para seleção de participantes em pesquisas e para avaliação dos resultados obtidos por meio da intervenção.

O critério de inclusão para participação das crianças e adolescentes nas pesquisas foi o de terem diagnóstico de $\mathrm{TB}$, mas não informaram com precisão como foi 
realizado e incluíram outros que se diferenciaram para seleção da amostra. Dentre os 21 estudos avaliados, seis incluíram como critério de seleção a ocorrência de pelo menos um episódio maníaco com exaltação e grandiosidade, misto, hipomaníaco ou depressivo em até seis meses anteriores à intervenção. Em seis dos estudos foi exigida a participação efetiva de um dos responsáveis. Em quatro estudos as crianças deveriam apresentar estabilidade em relação aos efeitos farmacológicos e o interesse em permanecer submetendo-se ao tratamento medicamentoso. Em dois foi aplicado um teste de quociente de inteligência e exigida pontuação mínima para participação, sendo que um deles exigiu também uma determinada pontuação nas escalas Young Mania Rating Scale (YMRS) ou Childrens Depression Rating Scale - Revised (CDRS-R).

Nas intervenções descritas pode-se constatar a existência de algumas medidas de avaliação tais como, inventários, testes psicométricos e escalas que, embora não tenham sido desenvolvidas para o TB em crianças e adolescentes, estão sendo empregadas com frequência. Após a análise dos estudos, constatou-se que dentre as 21 pesquisas avaliadas, 18 recorreram a essas medidas de avaliação como pré e/ou pós-testes. A utilização dessas medidas, apesar de ainda não ser o ideal em relação à avaliação, tem aparecido como uma tentativa de proporcionar validade e efetividade para as pesquisas. Esse é um fato relevante, uma vez que tem contribuído para maior controle nos estudos e busca por fidedignidade.

Apesar de algumas limitações metodológicas que foram encontradas nos estudos revisados, resultados efetivos são apresentados e mostram que a intervenção psicossocial é importante para auxiliar pacientes e suas famílias a conviverem melhor com o transtorno e consequentemente diminuir possíveis prejuízos sociais causados por ele, mesmo quando os sintomas não estão presentes. No entanto, considerase pequena a quantidade de pesquisas que a utiliza com crianças e adolescentes com TB. Isso pode acontecer devido às dificuldades enfrentadas na definição e caracterização do TB para esta população como mencionado ao longo do artigo. Por esta razão, a continuidade de pesquisas desenvolvidas nessa área poderá contribuir para a elaboração de programas de intervenção psicossocial direcionados especificamente para essas famílias.

\section{Referências}

Angst, J. (2013). Bipolar disorders in DSM-5: strengths, problems and perspectives. International Journal of Bipolar Disorders, 1(1), 1-3. http://dx.doi.org/10.1186/2194-7511-1-12

Apps J., Winkler J., \& Jandrisevits, M. D. (2008). Bipolar disorders: Symptoms and treatment in children and adolescents. Pediatric Nursing, 34, 84-88.

Axelson, D., Goldstein, B., Goldstein, T., Monk, K., Yu, H., Hickey, M. B., ... \& Iyengar, S. (2015). Diagnostic precursors to bipolar disorder in offspring of parents with bipolar disorder: a longitudinal study. American Journal of Psychiatry, 172(7), 638-646. http://dx.doi.org/10.1176/appi.ajp.2014.14010035

Belzeaux, R., Boyer, L., Mazzola-Pomietto, P., Michel, P., Correard, N., Aubin, V., ... \& Etain, B. (2015). Adherence to medication is associated with non-planning impulsivity in euthymic bipolar disorder patients. Journal of Affective Disorders, 184, 60-66. http://dx.doi.org/10.1016/j.jad.2015.05.041

Birmaher, B. (2013). Bipolar disorder in children and adolescents. Child and adolescent mental health, 18(3), 140-148. http://dx.doi.org/10.1111/camh.1202

Brotman, M., Schmajuk, M., Rich, B., Dickstein, D. P., Guyer, A., \& Costello, E. J. (2006). Prevalence, clinical correlates and longitudinal course of severe mood dysregulation in children. Biological Psychiatry, 60(9), 991-997. http://dx.doi. org/10.1016/j.biopsych.2006.08.042

Carr, A. (2009). Bipolar disorder in younger people: Description, assessment and evidence-based treatment. Developmental Neurorehabilitation, 12(6), 427-441. http://dx.doi.org/10.3109/17518420903042454

Colom, F., \& Vieta, E. (2004). Melhorando o desfecho do transtorno bipolar usando estratégias não farmacológicas: O papel da psicoeducação. Revista Brasileira de Psiquiatria, 26(3), 47-50. http://dx.doi.org/10.1590/S151644462004000700011

Crump, C., Winkleby, M. A., Sundquist, K., \& Sundquist, J. (2014). Comorbidities and mortality in persons with schizophrenia: a Swedish national cohort study. American Journal of Psychiatry, 170(3), 324-333. http://dx.doi. org/10.1176/appi.ajp.2012.12050599

Danielson, C. K., Feeny, N. C., Findling, R. L., \& Youngstrom, E. A. (2004). Psychosocial treatment of bipolar disorders in adolescents: A proposed cognitive-behavioral intervention. Cognitive and Behavioral Practice, 11, $283-297$. http://dx.doi.org/10.1016/S1077-7229(04)80043-9 
Dusetzina, S. B., Weinberger, M., Gaynes, B. N., Farley, J. F., Sleath, B., \& Hansen, R. A. (2012). Prevalence of bipolar disorder diagnoses and psychotropic drug therapy among privately insured children and adolescents. Pharmacotherapy: The Journal of Human Pharmacology and Drug Therapy, 32(12), 1085-1094. http://dx.doi.org/10.1002/phar.1148

Fagiolini, F. E., Axelson, D. A., Birmaher, B., Cheng, Y., Curet, D. E ... Kupfer, D. J. (2009). Enhancing outcomes in patients with bipolar disorder: Results from the Bipolar Disorder Center for Pennsylvanians Study. Bipolar Disorder, 11(4), 382-390.. http://dx.doi.org/10.1111/j.1399-5618.2009.00700.x

Feeny, N. C., Danielson, C. K., Schwartz, L., Youngstrom, E. A., \& Findling, R. L. (2006). Cognitive behavioral therapy for bipolar disorders in adolescents: A pilot study. Bipolar Disorder, 8, 508-515. http://dx.doi.org/10.1111/j.13995618.2006.00358.x

Frías, Á., Palma, C., \& Farriols, N. (2015). Comorbidity in pediatric bipolar disorder: Prevalence, clinical impact, etiology and treatment. Journal of Affective Disorders, 174, 378-389. http://dx.doi.org/10.1016/j.jad.2014.12.008

Fristad, M. A. (2006). Psychoeducational treatment for school-aged children with bipolar disorder. Development and Psychopathology, 18, 1289-1306. http://dx.doi.org/10.1017/S0954579406060627

Fristad, M. A., Davidson, K. H., \& Leffler, J. (2007). Thinking-feeling-doing: A therapeutic technique for children with bipolar disorder and their parents. Journal of Family Psychotherapy, 18(4), 81-104. http://dx.doi.org/10.1300/ J085v18n04 06

Fristad, M. A., Gavazzi, S. M., \& Koons, B. M. (2003). Family psychoeducation: An adjunctive intervention for children with bipolar disorder. Biological Psychiatry, 53(4), 382-390. http://dx.doi.org/10.1016/S0006-3223(03)00186-0

Fristad, M. A., Verducci, J. S., Walters, K., \& Young, M. E. (2009). Impact of multifamily psychoeducational psychotherapy in treating children aged 8 to 12 years with mood disorders. Archives of General Psichiatry, 66(9), 1013-1021. http://dx.doi.org/10.1001/archgenpsychiatry.2009.112

Fristad, M., Goldberg-Arnold, J., \& Gavazzi, S. (2002). Multi-family psychoeducation groups (MFPG) for families of children with bipolar disorder. Biological Psychiatry, 46(4), 254-262. http://dx.doi.org/10.1001/ archgenpsychiatry.2009.112

Geddes, J. R. \& Miklowitz, D. J. (2013). Treatment of bipolar disorder. The Lancet, 381(9878), 1672-1682. http://dx.doi. org/10.1016/S0140-6736(13)60857-0

Gibson, S., Brand, S. L., Burt, S., Boden, Z. V., \& Benson, O. (2013). Understanding treatment non-adherence in schizophrenia and bipolar disorder: a survey of what service users do and why. BMC psychiatry, 13(1), 153. http:// dx.doi.org/10.1186/1471-244X-13-153

Goldstein, B. I. (2012). Recent progress in understanding pediatric bipolar disorder. Archives of Pediatrics \& Adolescent Medicine, 166(4), 362-371. http://dx.doi.org/10.1001/archpediatrics.2011.832

Goldstein, B. I., Goldstein, T. R., Collinger, K. A., Axelson, D. A., Bukstein, O. G., Birmaher, B., \& Miklowitz, D. J. (2014b). Treatment development and feasibility study of family-focused treatment for adolescents with bipolar disorder and comorbid substance use disorders. Journal of psychiatric practice, 20(3), 237. http://dx.doi.org/10.1097/01. pra.0000450325.21791.7e

Goldstein, T. R., Axelson, D. A., Birmaher, B., \& Brent, D. A. (2010). Dialectical behavior therapy for adolescents with bipolar disorder: A 1-year open trial. Journal of the American Academy of Child and Adolescent Psychiatry, 46(7), 820-830. http://dx.doi.org/10.1097/chi.0b013e31805c1613

Goldstein, T. R., Fersch-Podrat, R., Axelson, D. A., Gilbert, A., Hlastala, S. A.; Birmaher, B., \& Frank, E. (2014a). Early intervention for adolescents at high risk for the development of bipolar disorder: Pilot study of Interpersonal and Social Rhythm Therapy (IPSRT). Psychotherapy, 51(1), 180-189. http://dx.doi.org/10.1037/a0034396

Goldstein, T. R., Goldstein, B. I., Montz, M. B., Bailey, B., \& Donaihy, A. (2011). A brief motivational intervention for preventing medication-associated weight gain among youth with bipolar disorder: Treatment development and case report. Journal of Child and Adolescent Psychopharmacology, 21(3), 275-280. http://dx.doi.org/10.1089/ cap.2010.0104

Grande, I., Berk, M., Birmaher, B., \& Vieta, E. (2015). Bipolar disorder. The Lancet (in press). http://dx.doi.org/10.1016/ S0140-6736(15)00241-X

Hlastala, S. A., Kotler, J. S., McClellan, J. M., \& McCauley, E. A. (2010). Interpersonal and social rhythm therapy for adolescents with bipolar disorder: Treatment development and results from and open trial. Depress Anxiety, 27(5), 457-464. http://dx.doi.org/10.1002/da.20668

Jairam, R., Srinath, S., Girimaji, S. C., \& Seshadri, S. P. (2004). A prospective 4-5 year follow-up of juvenile onset bipolar disorder. Bipolar Disorders, 6(5), 386-394. http://dx.doi.org/10.1111/j.1399-5618.2004.00149.x

Leclerc, E., Mansur, R. B., \& Brietzke, E. (2013). Determinants of adherence to treatment in bipolar disorder: a comprehensive review. Journal of affective disorders, 149(1), 247-252. http://dx.doi.org/10.1016/j.jad.2013.01.036

Leffler, J. M., Fristad, M. A., \& Klaus, N. M. (2010). Psychoeducational Psychotherapy (PEP) for children with bipolar disorder: Two case studies. Journal of Family Psychoterapy, 21, 269-286. http://dx.doi.org/10.1080/08975353. 2010.529051

Leibenluft, E. (2011). Severe mood dysregulation, irritability, and the diagnostic boundaries of bipolar disorder in youths. American Journal of Psychiatry, 168(2), 129-142. http://dx.doi.org/10.1176/appi.ajp.2010.10050766 
Miklowitz, D. J., Axelson, D. A., Birmaher, B., George, E. L., Taylor, D. O. et al. (2008). Family-focused treatment for adolescents with bipolar disorder results of a 2-year randomized trial. Archives of General Psychiatry, 65(9), 1053-1061. http://dx.doi.org/10.1001/archpsyc.65.9.1053

Miklowitz, D. J., Chang, K. D., Taylor, D. O., George, E. L., Singh, M. K., Schneck, C. D. et al. (2011). Early psychosocial intervention for youth at risk for bipolar I or II disorder: A one-year treatment development trial. Bipolar Disorder, 13(1), 67-75. http://dx.doi.org/10.1111/j.1399-5618.2011.00890.x

Miklowitz, D. J., George, E. L., Axelson, D. A., Kim, E. Y., Birmaher, B., Schneck, C. et al. (2004). Family-focused treatment for adolescents with bipolar disorder. Journal of Affective Disorders, 82, 5113-5128. http://dx.doi. org/10.1016/j.jad.2004.05.020

Miklowitz, D. J., George, E. L., Richards, J. A., Simoneau, T. L., \& Suddath, R. L. (2003). A randomized study of familyfocused psychoeducation and pharmacotherapy in the outpatient management of bipolar disorder. Archives of General Psychiatry, 60(9), 904-912. http://dx.doi.org/10.1001/archpsyc.60.9.904

Miklowitz, D. J., Schneck, C. D., George, E. L., Taylor, D. O., Sugar, C. A., Birmaher, B., ... \& Axelson, D. A. (2014). Pharmacotherapy and Family-Focused Treatment for Adolescents With Bipolar I and II Disorders: A 2-Year Randomized Trial. American Journal of Psychiatry, 171(6), 658-667. http://dx.doi.org/10.1176/appi.ajp.2014.13081130

Miller, S., Chang, K. D., \& Ketter, T. A. (2013). Bipolar disorder and attention-deficit/hyperactivity disorder comorbidity in children and adolescents: evidence-based approach to diagnosis and treatment. The Journal of Clinical Psychiatry, 74(6), 628. http://dx.doi.org/10.4088/JCP.13ac08565

Morken, G., Vaaler, A. E., \& Folden, E. G. (2009). Age at onset of first episode and time to treatment in in-patients with bipolar disorder. British Journal of Psychiatry, 194, 559-560. http://dx.doi.org/10.1192/bjp.bp.108.054452

Organização Mundial da Saúde [OMS] (1992). Classificação estatística internacional de doenças (10â ed.). São Paulo: EDUSP.

Pacheco, H. A., Fratoni, M. M., Parcias, S. R., Leal, B. M., da Silva, M. E. M., Guimarães, A. C. A., \& Boing, L. (2014). Levantamento de manifestações clínicas na infância e adolescência de fatores precoces do transtorno bipolar. Pediatria Moderna, 50(4).

Pavuluri, M. N., Graczyk, P. A., Henry, D. B., Carbray, J. A., Heidenreich, J., \& Miklowitz, D. J. (2004). Child- and family-focused cognitive-behavioral therapy for pediatric bipolar disorder: Development and preliminary results. Journal of the Academy of Child \& Adolescent Psychiatry, 43(5), 528-537. http://dx.doi.org/10.1097/00004583200405000-00006

Phillips, M. L. \& Kupfer, D. J. (2013). Bipolar disorder diagnosis: challenges and future directions. The Lancet, 381(9878), 1663-1671. http://dx.doi.org/10.1016/S0140-6736(13)60989-7

Prien R. F. \& Rush A. J. (1996). National Institute of Mental Health workshop report on the treatment of bipolar disorder. Biological Psychiatry, 40, 215-220. http://dx.doi.org/10.1016/0006-3223(95)00379-7

Ramires, V. R. R., Benetti, S. P. C., Silva, F. J. L., \& Flores, G. G. (2009). Saúde mental de crianças no Brasil: Uma revisão de literatura. Interação em Psicologia, 13(2), 311-322.

Reinares, M., Sánchez-Moreno, J., \& Fountoulakis, K. N. (2014). Psychosocial interventions in bipolar disorder: what, for whom, and when. Journal of affective disorders, 156, 46-55. http://dx.doi.org/10.1016/j.jad.2013.12.017

Serrano E., Ezpeleta L., Alda J. A., Matalí J. L., \& San L. (2011). Psychometric properties of the Young Mania Rating Scale for the identification of mania symptoms in Spanish children and adolescents with attention deficit/hyperactivity disorder. Psychopathology, 11(3), 125-132. http://dx.doi.org/10.1159/000320893

Severus, E. \& Bauer, M. (2013). The impact of treatment decisions on the diagnosis of bipolar disorders. International Journal of Bipolar Disorders, 2, 3-3. http://dx.doi.org/10.1186/2194-7511-2-3

Sheriff, R. J., McGorry, P. D., Cotton, S., \& Yung, A. R. (2015). A Qualitative Study of the Prodrome to FirstEpisode Major Depressive Disorder in Adolescents. Psychopathology, 48(3), 153-161. http://dx.doi.org/10.1159/ 000373894

Van Meter, A. R., Burke, C., Kowatch, R. A., Findling, R. L., \& Youngstrom, E. A. (2016). Ten-year updated metaanalysis of the clinical characteristics of pediatric mania and hypomania. Bipolar disorders (in press). http://dx.doi. org/10.1111/bdi.12358

Van Meter, A. R., Moreira, A. L., \& Youngstrom, E. A. (2011). Meta-analysis of epidemiologic studies of pediatric bipolar disorder. The Journal of Clinical Psychiatry, 72(9), 1250-1256. http://dx.doi.org/10.4088/JCP.10m06290

West, A. E, \& Pavuluri, M. N. (2009). Psychosocial treatment for childhood and adolescent bipolar disorder. Child and Adolescent Psychiatric Clinics of North America, 18, 471-482. http://dx.doi.org/10.1016/j.chc.2008.11.009

West, A. E., Jacobs, R. H., Westerholm, R., Lee, A., Carbray, J., Heidenreich, J., \& Pavuluri, M. N. (2009). Child and family-focused cognitive-behavioral therapy for pediatric bipolar disorder: Pilot study of group treatment format. Journal of the Canadian Academy of Child and Adolescent Psychiatry, 18(3), 239-246.

Yen, S., Stout, R., Hower, H., Killam, M. A., Weinstock, L. M., Topor, D. R., ... \& Goldstein, B. I. (2015). The influence of comorbid disorders on the episodicity of bipolar disorder in youth. Acta Psychiatrica Scandinavica (in press). http:// dx.doi.org/10.1111/acps. 12514 
Young, S., Pfaff, D., Lewandowski, K. E., Ravichandran, C., Cohen, B. M., \& Öngür, D. (2013). Anxiety disorder comorbidity in bipolar disorder, schizophrenia and schizoaffective disorder. Psychopathology, 46(3), 176-185. http://dx.doi.org/10.1159/000339556

Zazula, R. \& Gon, M. C. C. Instruções Verbais e o Seguir Recomendações Médicas para Tratamento da Dermatite Atópica na Infância. Interação em Psicologia, 18(2), 155-165. http://dx.doi.org/10.5380/psi.v18i2.29589

Autores:

Renata Garcia de Almeida Moraes - Mestre, Centro Universitário Filadélfia

Márcia Cristina Caserta Gon - Doutor, Universidade Estadual de Londrina.

Robson Zazula - Mestre, Universidade Federal da Integração Latino Americana.

Endereço para correspondência:

<robson.zazula@unila.edu.br>

Recebido em: 24.02.2015

Aceito em: 25.01.2016 